\title{
Frailty syndrome: an overview
}

This article was published in the following Dove Press journal:

Clinical Interventions in Aging

19 March 2014

Number of times this article has been viewed

\section{Xujiao Chen' \\ Genxiang Mao' \\ Sean $X$ Leng $^{2}$}

'Department of Geriatrics, Zhejiang Hospital, Hangzhou, People's

Republic of China; ${ }^{2}$ Division of Geriatric Medicine and Gerontology,

Department of Medicine, Johns Hopkins University School of Medicine, Baltimore, MD, USA
Correspondence: Sean X Leng

Division of Geriatric Medicine and Gerontology, Department of Medicine, Johns Hopkins University School of Medicine, 550I Hopkins Bayview Circle - Room IA.38A, Baltimore, MD 21224, USA

Tel +I 4105502494

Fax + I 4I05503/43

Email sleng5@jhmi.edu
Abstract: Frailty is a common and important geriatric syndrome characterized by age-associated declines in physiologic reserve and function across multiorgan systems, leading to increased vulnerability for adverse health outcomes. Two major frailty models have been described in the literature. The frailty phenotype defines frailty as a distinct clinical syndrome meeting three or more of five phenotypic criteria: weakness, slowness, low level of physical activity, self-reported exhaustion, and unintentional weight loss. The frailty index defines frailty as cumulative deficits identified in a comprehensive geriatric assessment. Significant progress has recently been made in understanding the pathogenesis of frailty. Chronic inflammation is likely a key pathophysiologic process that contributes to the frailty syndrome directly and indirectly through other intermediate physiologic systems, such as the musculoskeletal, endocrine, and hematologic systems. The complex multifactorial etiologies of frailty also include obesity and specific diseases. Major clinical applications include risk assessment and stratification. This can be applied to the elderly population in the community and in a variety of care settings. Frailty may also be useful for risk assessment in surgical patients and those with cardiovascular diseases, cancer, or human immunodeficiency virus infection, as well as for assessment of vaccine effectiveness in older adults. Currently, exercise and comprehensive geriatric interdisciplinary assessment and treatment are key interventions for frailty. As understanding of the biologic basis and complexity of frailty further improves, more effective and targeted interventional strategies and innovative geriatric-care models will likely be developed.

Keywords: frailty, inflammation, IL-6, aging, older adults

\section{Introduction}

Identification of older individuals who are frail or at risk of becoming frail with appropriate subsequent evaluation and intervention constitutes a cornerstone of geriatric medicine and quality care for the ever-growing elderly population. ${ }^{1-3}$ While health care providers and researchers in the field of aging have long been aware of the term of "frailty", defining this syndrome proved to be elusive until recently. Geriatricians used to say, "I know it when I see it, but what I see may not be the same as what everyone else sees." Impressive progress has been made in the past decade also, and the number of scientific publications on this topic has grown exponentially. Efforts have also been made internationally to reach frailty consensus. This article provides an overview of the current state of our knowledge about the frailty syndrome: its definitions and pathogenesis, as well as clinical applications and potential preventative and therapeutic interventions. 


\section{Definitions}

Frailty is conceptually defined as a clinically recognizable state of older adults with increased vulnerability, resulting from age-associated declines in physiologic reserve and function across multiple organ systems, such that the ability to cope with everyday or acute stressors is compromised. ${ }^{1,4-7}$ Based on this conceptual framework, two major definitions with proposed assessment tools have emerged over the past decade also: the frailty phenotype (FP), also known as Fried's definition or Cardiovascular Health Study (CHS) definition, ${ }^{5}$ and the frailty index (FI). ${ }^{8}$ A number of other definitions have also been described in the literature, including FRAIL (Fatigue, Resistance, Ambulation, Illnesses, Loss of weight) (International Academy of Nutrition and Aging), ${ }^{9}$ Frailty Instrument for Primary Care of the Survey of Health, Ageing and Retirement in Europe (SHARE-FI), ${ }^{10}$ and the Groningen Frailty Indicator. ${ }^{11}$ Major international efforts have recently been made to reach frailty consensus. ${ }^{12}$ Although no single operational definition or simple assessment tool has been agreed upon, a consensus has been established that frailty 1) is a clinical syndrome, 2) indicates increased vulnerability to stressors, leading to functional impairment and adverse health outcomes, 3) might be reversible or attenuated by interventions, and 4) is useful in primary care. ${ }^{12}$ This review will focus on FP and FI.

\section{Frailty phenotype}

The phenotypic definition of frailty as a geriatric syndrome was proposed by Fried et al and tested in the CHS, a largecohort study of over 5,300 community-dwelling older men and women in the US. ${ }^{5,13}$ Frailty is operationalized as a syndrome meeting three or more of five phenotypic criteria: weakness as measured by low grip strength, slowness by slowed walking speed, low level of physical activity, low energy or self-reported exhaustion, and unintentional weight loss (Table 1). A prefrail stage, in which one or two criteria

Table I The frailty phenotype (FP)

\begin{tabular}{|c|c|}
\hline FP criteria & Measurement \\
\hline Weakness & $\begin{array}{l}\text { Grip strength: lowest } 20 \% \\
\text { (by sex, body mass index) }\end{array}$ \\
\hline Slowness & $\begin{array}{l}\text { Walking time/ } 15 \text { feet: slowest } 20 \% \\
\text { (by sex, height) }\end{array}$ \\
\hline Low level of physical activity & $\begin{array}{l}\text { Kcal/week: lowest } 20 \% \\
\text { Males: } 383 \mathrm{Kcal} / \text { week } \\
\text { Females: } 270 \mathrm{Kcal} / \text { week }\end{array}$ \\
\hline Exhaustion; poor endurance & “Exhaustion” (self-report) \\
\hline Weight loss & $\begin{array}{l}>10 \text { lb lost unintentionally in } \\
\text { prior year }\end{array}$ \\
\hline
\end{tabular}

Note: Adapted from Fried LP, Tangen C, Walston J, et al. Frailty in older adults: evidence for a phenotype. J Gerontol A Biol Sci Med Sci. 200I;56A:MI-MII, by permission of Oxford University Press. ${ }^{5}$ are present, identifies a subset at high risk of progressing to frailty. Older individuals with none of the above five criteria are classified as nonfrail. ${ }^{5}$ This definition recognizes frailty as a distinct clinical entity distinguished from disability, as measured by impairment in activities of daily living (ADL) and comorbidity defined by two or more diseases; two other prevalent conditions in older adults. ${ }^{5,14}$ All three conditions are predictive in varying degrees of adverse health outcomes, and therefore have a certain level of overlap (Figure 1). However, the main features of frailty, such as decreased functional reserve, impairment or dysregulation in multiple physiological systems, and reduced ability to regain physiological homeostasis after a stressful and destabilizing event, make the distinction of frailty from disability or comorbidity relatively easy. Disability suggests chronic limitations or dependence in mobility and/or ADL or instrumental ADL. While many (but not all) frail individuals are disabled, not all disabled persons are frail. For example, older patients who suffer severe disability secondary to a major accident or stroke may maintain relatively intact function in other physiological systems, and thus are not frail. Comorbidity indicates the presence of multiple chronic diseases. Not surprisingly, comorbidity is associated with increased risk of adverse clinical outcomes, as evidenced by higher short-term and long-term mortality and significantly increased physical disability compared with those without diseases. However, the mere presence of two or more clinical diagnoses in itself may not identify the vulnerable group of older patients or those who are frail. When comorbid conditions worsen, are

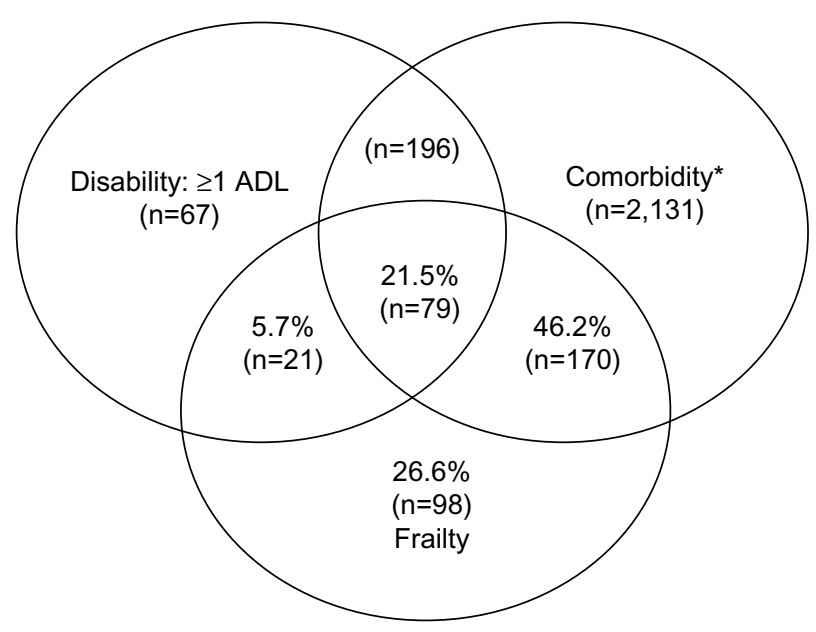

Figure I Venn diagram of the frailty syndrome, activities of daily living (ADL) disability, and comorbidity (two or more diseases) in the Cardiovascular Health Study dataset, demonstrating frailty as a distinct geriatric syndrome with some overlap with disability and comorbidity. Fried LP, Tangen C, Walston J, et al. Frailty in older adults: evidence for a phenotype. J Gerontol A Biol Sci Med Sci. 200I;56A:MI-MII, by permission of Oxford University Press. ${ }^{5}$

Note: *Two or more out of the following nine diseases: myocardial infarction, angina, congestive heart failure, claudication, arthritis, cancer, diabetes, hypertension, COPD. 
not adequately treated, and/or more diseases are accumulated, these patients may develop frailty. ${ }^{14}$ Nevertheless, diseases and disability are important confounding factors that deserve careful consideration in frailty assessment.

To test the internal validity of the FP, Bandeen-Roche et al analyzed the data from a combined sample of women aged 70-79 years from the Women's Health and Aging Studies (WHAS) I and II and identified such patterns of cooccurrence of the five phenotypic criteria as manifestation in a critical mass and aggregation in a hierarchical order, as would occur in a cycle in which dysregulation in a sentinel system may trigger a cascade of alterations across other systems. These findings support the internal validity of the FP vis-à-vis the stated theory characterizing frailty as a clinical syndrome, and provide justification for the current counting strategy for defining frailty categories (ie, nonfrail, prefrail, and frail). ${ }^{15}$ Various adaptations of this definition have been described in the literature, and were often based upon available measures in specific studies rather than meaningful conceptual differences.

Based on the FP and its various modified versions, the overall prevalence of frailty in community-dwelling older adults aged 65 years and over in the US ranges from 7\% to $12 \%$. It increases with age from $3.9 \%$ in the age-group $65-74$ years to $25 \%$ in the age group older than 85 years. ${ }^{14}$ The prevalence of frailty is higher in women than in men ( $8 \%$ versus 5\%) and higher in African Americans than in Caucasians (13\% versus $6 \%$ in the CHS and $16 \%$ versus $10 \%$ in the WHAS). It also appears to have geographic differences. The overall prevalence is $17 \%$ in Europe, ranging from 5.8\% in Switzerland to $27 \%$ in Spain, while the prevalence in Latin American and Caribbean cities is much higher, ranging from $30 \%$ to $48 \%$ in women and from $21 \%$ to $35 \%$ in men. ${ }^{16,17}$

\section{Frailty index}

The FI was developed by Rockwood et al based on a comprehensive geriatric assessment by counting the number of deficits accumulated, including diseases, physical and cognitive impairments, psychosocial risk factors, and common geriatric syndromes other than frailty. ${ }^{8,18}$ The criteria for a variable to be considered as a deficit are that the variable needs to be acquired, age-associated, associated with an adverse outcome, and should not saturate too early. The last criterion means that the proportion of older adults who have the deficit should not be close to $100 \%$, because the deficit is uninformative at that point. For example, nocturia, although it is age-associated, disrupts sleep, and is a deficit, cannot be counted in the FI, because it is so common that it is typically seen in more than $90 \%$ of men 75 years or older. ${ }^{18}$ The total number of deficits that can be used in the FI is considered to be 80 , with $30-70$ items being typically counted. ${ }^{19}$

Compared to the FP definition described earlier, the FI appears to be a more sensitive predictor of adverse health outcomes, because of its more finely graded risk scale and inclusion of deficits that likely have causal relationships with adverse clinical outcomes. ${ }^{20}$ While the FI may have clinical utility in risk assessment and stratification, it is not clear if it adds significant value to comprehensive geriatric assessment. In addition, the FI does not attempt to distinguish frailty from disability or comorbidity. Instead, it includes them or their associated deficits. Moreover, counting the number of accumulated deficits does not constitute a clinical geriatric syndrome per se. As such, the FI makes it difficult, if not impossible, to further investigate underlying mechanisms and etiology of frailty. Therefore, recent advances in the pathogenesis of frailty described in the following section are almost exclusively based on the FP definition.

\section{Pathogenesis}

Frailty is characterized by multisystem dysregulations, leading to a loss of dynamic homeostasis, decreased physiologic reserve, and increased vulnerability for subsequent morbidity and mortality. This is often manifested by maladaptive response to stressors, leading to a vicious cycle toward functional decline and other serious adverse health outcomes. ${ }^{1,4-7,21}$ A large body of literature, most rapidly accumulated in the past few years, suggests several important multisystem pathophysiologic processes in the pathogenesis of the frailty syndrome, including chronic inflammation and immune activation, and those in musculoskeletal and endocrine systems. Chronic inflammation is likely a key underlying mechanism that contributes to frailty directly and indirectly through other intermediate pathophysiologic processes (Figure 2). Potential etiologic factors include genetic/epigenetic and metabolic factors, environmental and lifestyle stressors, and acute and chronic diseases. This section provides a brief overview of our current understanding of each of these key pathophysiologic processes.

\section{Chronic inflammation and immune activation}

Direct association between frailty and elevated circulating levels of interleukin (IL)-6, a proinflammatory cytokine, was first observed in community-dwelling older adults. ${ }^{22} \mathrm{~A}$ large number of studies in many cohorts of older adults and under various care settings have since provided evidence supporting the role of chronic inflammation and immune activation in 


\section{Etiology/risk factors}

Potential mechanisms

Chronic inflammation
Frailty
phenotype

Intermediary

systems

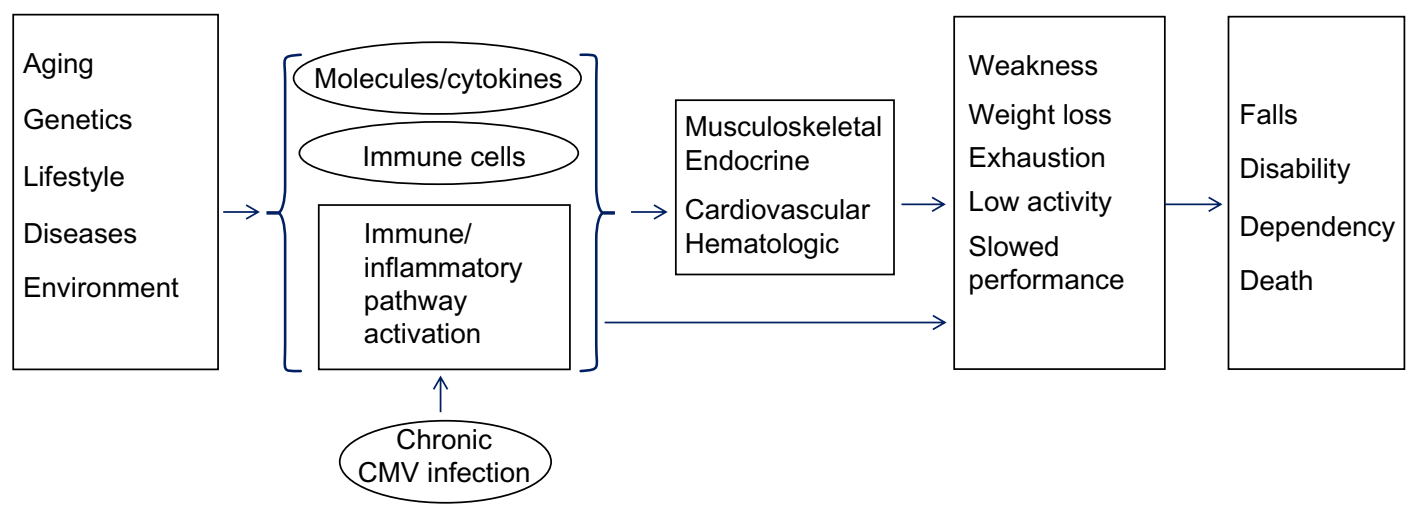

Figure 2 Pathogenesis of the frailty syndrome: current understanding of potential underlying mechanisms and hypothetical modal pathways leading to frailty. Abbreviation: CMV, Cytomegalovirus.

the pathogenesis of the frailty syndrome..$^{23-25}$ While in-depth review of this active area of research is beyond the scope of this overview, several lines of scientific evidence should be emphasized here.

\section{Molecular markers of chronic inflammation and immune activation in frailty}

Subsequent studies in cell culture, mouse model, and large older-adult cohorts have confirmed the association between elevated IL-6 levels and frailty. ${ }^{26-30}$ Other inflammatory molecules, including C-reactive protein and tumor necrosis factor- $\alpha$, have also been shown to have elevated levels in frail older adults. ${ }^{29-31}$ Moreover, elevated levels of neopterin, a well-known molecular marker for immune activation mediated by monocytes and macrophages, ${ }^{32,33}$ are associated with frailty in community-dwelling older adults independently of IL-6 levels, suggesting that immune activation can potentially be a preceding process leading to chronic inflammation in the pathogenesis of frailty.

\section{Cellular components of inflammatory/immune system and pathway activation in frailty}

Drastic increase (above the normal range) of total white blood cell (WBC) count, which is routinely measured as part of the complete blood counts in clinical practice, is recognized as a laboratory indicator for systemic inflammation frequently secondary to acute bacterial infections. Recent studies have demonstrated direct associations between frailty and increased total WBC count, albeit still under the upper limit of the normal range, and counts of its specific subpopulations including neutrophils and monocytes. ${ }^{28,34}$
In the T-lymphocyte subpopulation, frailty is associated with increased counts of cluster of differentiation (CD) $8^{+}$ CD28- $\mathrm{T}$ cells and $\mathrm{CCR}^{+} \mathrm{T}$ cells, the latter of which has a type 1 proinflammatory phenotype. ${ }^{35-37}$ A recent study of inflammatory pathway activation through in-depth analyses of pathway-specific gene expression by purified monocytes from frail older individuals has shown upregulation in ex vivo expression of several stress-responsive inflammatory pathway genes in frailty. ${ }^{38}$ This inflammatory pathway activation as a molecular mechanism leading to chronic inflammation in the frailty syndrome is suggested by the observed correlation between frailty-associated upregulated monocytic expression of CXCL10, a potent proinflammatory chemokine, and elevated circulating IL-6 levels. ${ }^{39}$ However, etiologies and mechanisms that contribute to immune and inflammatory pathway activation in frailty remain to be investigated. One possibility is chronic/persistent Cytomegalovirus (CMV) infection, as positive anti-CMV immunoglobulin $\mathrm{G}$ titers have been shown to be associated with frailty. ${ }^{40,41}$ In addition, chronic CMV infection, as defined by the presence of CMV deoxyribonucleic acid in peripheral blood monocytes, which distinguishes persistent from past CMV infections, is associated with expansion of $\mathrm{CMV}$-specific $\mathrm{CD} 8^{+} \mathrm{T}$ cells and elevated neopterin levels in older adults. ${ }^{42,43}$

\section{Direct and indirect contribution of chronic inflammation to frailty}

As discussed earlier, the relationship between frailty and common molecular and cellular inflammatory mediators is well documented. The critical question is whether chronic inflammation plays a role in the pathogenesis of frailty. 
Individual inflammatory molecules, such as IL-6, may directly contribute to frailty or its central components (such as decreased muscle mass, strength, and power, and slowed motor performance). ${ }^{28,44-46}$ As frailty involves multisystem physiologic dysregulation, it is conceivable that chronic inflammation contributes to frailty through its detrimental effects on other physiologic organ systems, such as musculoskeletal and endocrine systems (see following), anemia, clinical and subclinical cardiovascular diseases, and nutritional dysregulation. ${ }^{21,47,48}$ In fact, studies have shown that elevated cellular and molecular inflammatory mediators have inverse associations with hemoglobin concentrations, insulin-like growth factor (IGF)-1 levels, and levels of albumin, micronutrients, and vitamins. ${ }^{22,29,49-52}$ Given these factors, it has been proposed that the heightened inflammatory state plays a key role in the pathogenesis of frailty directly or indirectly through other intermediate pathophysiologic processes (Figure 2). However, factors other than chronic inflammation may also be important in the pathogenesis of frailty, as no consistent associations were observed between elevated IL-6 levels and prevalent or incident frailty in some studies $^{53,54}$ and the use of statins, which are known to have anti-inflammatory effects, had no association with reduction of incident frailty. ${ }^{55}$

\section{Musculoskeletal system}

Given that weakness and slowed motor performance are cardinal features of the frailty syndrome, sarcopenia is likely a key pathophysiologic contributor to frailty. In fact, investigators in Europe and Asia consider sarcopenia research a potentially useful initial step toward interventional studies of the frailty syndrome. ${ }^{56,57}$ Sarcopenia is defined as the loss of muscle mass and strength, which can occur rapidly after the age of 50 years. It can be further accelerated by chronic diseases, and is a major contributor to disability. Its causes include age-related changes in $\alpha$-motor neurons, type I muscle fibers, muscular atrophy, poor nutrition, growth hormone $(\mathrm{GH})$ production, sex-steroid levels, and physical activity. As discussed earlier, chronic inflammation is also an important contributor to sarcopenia. Skeletal muscle provides important support for bone health. The frailty syndrome has also been shown to have direct relationships with osteopenia and osteoporosis. ${ }^{1,4,5,58}$

\section{Endocrine system}

Sex steroids and IGF-1 are essential to skeletal muscle metabolic dysregulation. For example, age-related rapid decrease of estrogen in postmenopausal women and gradual decrease of testosterone in older men lead to decline in muscle mass and muscle strength. Circulating levels of the sex hormone dehydroepiandrosterone sulfate and IGF-1, a signaling target of GH, are significantly lower in frail than nonfrail older adults. ${ }^{46,49,50}$ Several other hormones, including cortisol and vitamin $\mathrm{D}$, have also been associated with the frailty syndrome in the elderly. For example, a positive association between higher levels of evening cortisol, 24-hour mean cortisol, and blunted diurnal variation of cortisol with frailty burden and clinical presentation has been observed in frail older women living in the community. ${ }^{59}$ In addition, recent findings from prospective cohort studies in older adults suggest that vitamin D insufficiency is associated with both prevalent and incident frailty, particularly in older men. ${ }^{46,60}$ Taken together, these studies suggest the potential role for dysregulations of the GH-IGF-1 somatotropic axis, the hypothalamic-pituitary-adrenal axis, and other hormones in the pathogenesis of frailty.

\section{Complex multifactorial etiology}

Figure 2 provides a simplified illustration of current understanding of the pathogenesis of the frailty syndrome. However, much remains to be learned about the complex multifactorial etiology of this geriatric syndrome. For example, Blaum et al showed significant association between obesity and frailty (defined by FP) in community-dwelling women aged 70-79 years. ${ }^{61}$ This association has been confirmed with FI and in other large-cohort studies. ${ }^{62,63}$ In addition, midlife obesity is shown to be predictive for the development of frailty in older men and women. ${ }^{64,65}$ Diseases may play an important etiologic role driving the development of frailty. They may also mimic the clinical manifestation of frailty. Moreover, acute episodes of illness or exacerbation of chronic conditions may accelerate the development of frailty or worsen its clinical presentation and adverse outcomes. Therefore, further clinical and biological investigations are needed to delineate the complex multifactorial etiology of frailty.

\section{Clinical applications}

As frailty is conceptualized as a vulnerable state associated with high risk for increased morbidity and mortality when exposed to a stressor, the frailty syndrome is considered a useful clinical tool for risk stratification in the highly heterogeneous elderly population. Evidence supporting this notion includes data from several large-cohort studies that demonstrated that frailty predicts increased falls, hospitalization, dependence, and mortality. $5,20,62,66$ The challenge is to develop a standardized frailty definition and screening 
tool that can be easily implemented in clinical practice. The European Union has placed special emphasis on defining frailty, as frail elders are high users of community resources, hospitalization, and nursing homes. A frailty consensus has recently been established through concerted international effort. ${ }^{12}$ It is hoped that this and other collaborative efforts will address the pressing challenge and make frailty a useful clinical assessment tool to identify those who are frail, so that targeted interventions can be developed to improve their health and quality of life as well as utilization of health care resources. In fact, promising results from such efforts in France were presented at the International Association of Gerontology and Geriatrics Global Aging Research Network symposium on "implementing frailty into clinical practice and clinical research" at the 20th World Congress of Gerontology and Geriatrics in June 2013.

Emerging evidence suggests that frailty is a useful risk assessment tool for preoperative evaluation in elderly patients who undergo surgery. Both the FI and FP have been shown to be predictive for increased postoperative complications. ${ }^{67,68}$ Frailty may also be useful for risk assessment in older patients with cardiovascular conditions, as it predicts increased morbidity and mortality in this elderly patient population, including patients undergoing cardiac surgery. ${ }^{69,70}$ Furthermore, frailty may be a clinical marker for overall immune functional decline in older adults, as the FP has been shown to identify those who fail to mount adequate immune responses to influenza and pneumococcal immunizations and are at high risk for these common infections and their complications. ${ }^{71,72}$

Frailty assessment may provide novel insight into heightened vulnerability and risk stratification of older patients with cancer. ${ }^{73,74}$ Therefore, the frailty syndrome constitutes a critical issue in geriatric oncology. This is because traditional tools, including chronological age and the Eastern Cooperative Oncology Group performance status, do not assess physiological reserve and have poor predictive value for poor clinical outcomes in this patient population. ${ }^{74,75}$ Frailty, on the other hand, has been shown to be predictive for postoperative complications among elderly patients with malignant gynecologic tumors. ${ }^{76}$ In addition, Aaldriks et al have demonstrated that the presence of frailty is highly predictive for increased mortality in older patients with advanced colorectal cancer receiving chemotherapy. ${ }^{77}$ Moreover, recent data have shown that frailty and prefrailty are common in breast cancer survivors and may occur at an earlier age in these survivors than in those with no history of breast cancer. ${ }^{78}$ Taken together, these studies suggest that frailty assessment is useful for risk stratification in geriatric oncology patients. Specific cancers may play an important etiologic role in the development of frailty.

Other areas of active research include human immunodeficiency virus (HIV) infection and aging. In two large-cohort studies of HIV infection, frailty or frailty-related phenotype was shown to have a significant association with mortality and accelerated immune-function deterioration. ${ }^{79-81}$ In addition, recent studies have shown associations between frailty and cognitive decline and dementia. ${ }^{82-84}$ While the key pathophysiologic processes in the frailty syndrome described herein, including chronic inflammation, likely have adverse impacts on the aging brain, terms like "the frail brain" or "cognitive frailty" are controversial, because they may not be easily distinguishable from mild cognitive impairment or dementia. Moreover, it is unclear whether these terms add significant values beyond mild cognitive impairment or dementia.

\section{Potential interventions}

Broadly speaking, interventions for the frailty syndrome should aim to 1) prevent, delay, reverse, or reduce the severity of frailty, and 2) prevent or reduce adverse health outcomes in those whose frailty is not reversible. Effective interventional strategies likely have large benefits for elderly individuals, their families, and the whole society.

To date, exercise is the interventional modality that has most consistently shown benefit in treating frailty and its key components. ${ }^{85-88}$ Exercise has physiologic impacts on almost all organ systems, particularly musculoskeletal, endocrine, and immune systems. A large number of trials (albeit validated FP or FI was often not used at baseline or follow-up) have demonstrated the positive impact of exercise intervention on key components of the frailty syndrome, including muscle strength and functional mobility. ${ }^{88,89}$ Nutritional intervention is another nonpharmacological modality that may correct nutritional deficits, including that of micronutrients, and address weight loss of the frailty syndrome. However, evidence supporting its efficacy is currently lacking, and vigorous clinical evaluation is needed.

Effects of a pharmacological approach in the treatment of the frailty syndrome have not been adequately evaluated. Such hormonal therapy as testosterone, while it improves muscle strength, has significant systemic side effects. ${ }^{90}$ Estrogen-replacement therapy in postmenopausal women also has an unfavorable safety profile. ${ }^{91}$ Friedlander et al reported that IGF-1 therapy had a beneficial impact on bone density, muscle strength, or physical function in elderly women with no clinical IGF-1 deficiency. ${ }^{92}$ Currently available 
anti-inflammatory agents, while not formally evaluated in clinical trials in treating the frailty syndrome, also have significant adverse effects, particularly in the elderly. While vitamin D and angiotensin-converting enzyme inhibitors have favorable pharmacological and safety profiles, their clinical utility in the prevention and treatment of frailty has yet to be investigated. ${ }^{93}$

Another important area of interventions is to prevent biological, socioeconomic, and environmental stressors and improve clinical outcomes in elderly patients whose frailty is not reversible. Comprehensive geriatric interdisciplinary assessment and treatment has been demonstrated to improve health outcomes in frail older adults. The overall objectives of this interventional modality are to improve physical and psychological function, reduce hospitalization and iatrogenic adverse events, develop adaptive strategies addressing disability and dependence, improve quality of life, and decrease early mortality in older adults. The interdisciplinary assessment and care team usually consists of a geriatrician, a gerontologically trained nurse, a social worker, a pharmacist, and occupational and physical therapists. Patient assessment includes data collection via detailed medical history, physical examination, and limited ancillary evaluation (laboratory and/or imaging), as well as a thorough discussion and synthesis of relevant psychosocial and medical data and environmental resources, followed by the formulation of treatment goals and management plans developed with the direct participation of the patient and caregivers.

In summary, exercise and comprehensive geriatric interdisciplinary assessment and treatment are the key interventions for the frailty syndrome at the present time. Given the complex nature of this geriatric syndrome, any single agent or approach targeted to one single organ system may not achieve optimal results. Multimodality strategies intervening in potential biological, sociobehavioral, and environmental stressors should be considered for the frail elderly. As understanding of the biologic basis of frailty further improves, more effective interventional strategies that target specific physiologic systems and innovative geriatric care models are likely to be developed.

\section{Acknowledgments}

Dr Genxiang Mao is an Irma and Paul Milstein Program for Senior Health fellow supported by the Milstein Medical Asian American Partnership (MMAAP) Foundation (http:// www.mmaapf.org). This work was also supported in part by NIH grant R21-AG-043874 to Dr Sean X Leng.

\section{Disclosure}

The authors report no conflicts of interest in this work.

\section{References}

1. Fried LP, Hadley EC, Walston JD, et al. From bedside to bench: research agenda for frailty. Sci Aging Knowledge Environ. 2005;2005(31):pe24.

2. Walston J, Hadley EC, Ferrucci L, et al. Research agenda for frailty in older adults: toward a better understanding of physiology and etiology: summary from the American Geriatrics Society/National Institute on Aging Research Conference on Frailty in Older Adults. J Am Geriatr Soc. 2006;54:991-1001.

3. Clegg A, Young J, Iliffe S, Rikkert MO, Rockwood K. Frailty in elderly people. Lancet. 2013;381:752-762.

4. Lipsitz LA. Dynamics of stability: the physiologic basis of functional health and frailty. J Gerontol A Biol Sci Med Sci. 2002;57:115-125.

5. Fried LP, Tangen C, Walston J, et al. Frailty in older adults: evidence for a phenotype. J Gerontol A Biol Sci Med Sci. 2001;56A:M1-M11.

6. Bortz WM. The physics of frailty. J Am Geriatr Soc. 1993;41: 1004-1008.

7. Lipsitz LA, Goldberger AL. Loss of 'complexity' and aging. Potential applications of fractals and chaos theory to senescence. JAMA. 1992;267:1806-1809.

8. Jones DM, Song X, Rockwood K. Operationalizing a frailty index from a standardized comprehensive geriatric assessment. J Am Geriatr Soc. 2004;52:1929-1933.

9. Abellan van KG, Rolland Y, Bergman H, Morley JE, Kritchevsky SB, Vellas B. The I.A.N.A Task Force on frailty assessment of older people in clinical practice. $J$ Nutr Health Aging. 2008;12:29-37.

10. Romero-Ortuno R, Walsh CD, Lawlor BA, Kenny RA. A frailty instrument for primary care: findings from the Survey of Health, Ageing and Retirement in Europe (SHARE). BMC Geriatr. 2010;10:57.

11. Peters LL, Boter H, Buskens E, Slaets JP. Measurement properties of the Groningen Frailty Indicator in home-dwelling and institutionalized elderly people. J Am Med Dir Assoc. 2012;13:546-551.

12. Morley JE, Vellas B, van Kan GA, et al. Frailty consensus: a call to action. J Am Med Dir Assoc. 2013;14:392-397.

13. Fried LP, Borhani NO, Enright P, et al. The Cardiovascular Health Study: design and rationale. Ann Epidemiol. 1991;1:263-276.

14. Fried LP, Ferrucci L, Darer J, Williamson JD, Anderson G. Untangling the concepts of disability, frailty, and comorbidity: implications for improved targeting and care. J Gerontol A Biol Sci Med Sci. 2004;59: 255-263.

15. Bandeen-Roche K, Xue QL, Ferrucci L, et al. Phenotype of frailty: characterization in the women's health and aging studies. J Gerontol A Biol Sci Med Sci. 2006;61:262-266.

16. Santos-Eggimann B, Cuenoud P, Spagnoli J, Junod J. Prevalence of frailty in middle-aged and older community-dwelling Europeans living in 10 countries. J Gerontol A Biol Sci Med Sci. 2009;64:675-681.

17. Alvarado BE, Zunzunegui MV, Beland F, Bamvita JM. Life course social and health conditions linked to frailty in Latin American older men and women. J Gerontol A Biol Sci Med Sci. 2008;63:1399-1406.

18. Searle SD, Mitnitski A, Gahbauer EA, Gill TM, Rockwood K. A standard procedure for creating a frailty index. BMC Geriatr. 2008;8:24.

19. Rockwood K, Mitnitski A. Frailty defined by deficit accumulation and geriatric medicine defined by frailty. Clin Geriatr Med. 2011;27: $17-26$.

20. Rockwood K, Mitnitski A. Frailty in relation to the accumulation of deficits. J Gerontol A Biol Sci Med Sci. 2007;62:722-727.

21. Fried LP, Xue QL, Cappola AR, et al. Nonlinear multisystem physiological dysregulation associated with frailty in older women: implications for etiology and treatment. J Gerontol A Biol Sci Med Sci. 2009;64:1049-1057.

22. Leng S, Chaves P, Koenig K, Walston J. Serum interleukin-6 and hemoglobin as physiological correlates in the geriatric syndrome of frailty: a pilot study. J Am Geriatr Soc 2002;50:1268-1271. 
23. Leng $\mathrm{S}$, Fried LP. Inflammatory markers and frailty. In: Fulop T, Franceschi C, Hirokawa K, Pawelec G, editors. Handbook on Immunosenescence: Basic Understanding and Clinical Applications. New York: Springer; 2009:1293-1303.

24. Yao X, Li H, Leng SX. Inflammation and immune system alterations in frailty. Clin Geriatr Med. 2011;27:79-87.

25. Li HF, Manwani B, Leng S. Frailty, inflammation, and immunity. Aging Dis. 2001;2:466-473.

26. Leng S, Yang H, Walston J. Decreased cell proliferation and altered cytokine production in frail older adults. Aging Clin Exp Res. 2004;16: 249-252.

27. Walston J, Fedarko N, Yang H, et al. The physical and biological characterization of a frail mouse model. J Gerontol A Biol Sci Med Sci. 2008;63:391-398.

28. Leng SX, Xue QL, Tian J, Walston JD, Fried LP. Inflammation and frailty in older women. J Am Geriatr Soc. 2007;55:864-871.

29. Hubbard RE, O'Mahony MS, Savva GM, Calver BL, Woodhouse KW. Inflammation and frailty measures in older people. $J$ Cell Mol Med. 2009;13:3103-3109.

30. Collerton J, Martin-Ruiz C, Davies K, et al. Frailty and the role of inflammation, immunosenescence and cellular ageing in the very old: cross-sectional findings from the Newcastle 85+ Study. Mech Ageing Dev. 2012;133:456-466.

31. Walston J, McBurnie MA, Newman A, et al. Frailty and activation of the inflammation and coagulation systems with and without clinical morbidities: results from the Cardiovascular Health Study. Arch Intern Med. 2002;162:2333-2341.

32. Leng SX, Tian X, Matteini A, et al. IL-6-independent association of elevated serum neopterin levels with prevalent frailty in communitydwelling older adults. Age Ageing. 2011;40:475-481.

33. Ramarathan R, Kohli A, Ingaramo MC, et al. Serum chitotriosidase, a putative marker of chronically activated macrophages, increases with normal aging. J Gerontol A Biol Sci Med Sci. 2013;68:1303-1309.

34. Leng SX, Xue QL, Tian J, Huang Y, Yeh SH, Fried LP. Association of neutrophil and monocyte counts with frailty in community-dwelling older women: results from the Women's Health and Aging Studies I. Exp Gerontol. 2009;44:511-516.

35. Semba RD, Margolick JB, Leng S, Walston J, Ricks MO, Fried LP. $\mathrm{T}$ cell subsets and mortality in older community-dwelling women. Exp Gerontol. 2005;40:81-87.

36. De FU, Wang GC, Fedarko NS, Walston JD, Casolaro V, Leng SX. T-lymphocytes expressing CC chemokine receptor-5 are increased in frail older adults. J Am Geriatr Soc. 2008;56:904-908.

37. Loetscher P, Uguccioni M, Bordoli L, et al. CCR5 is characteristic of Th1 lymphocytes. Nature. 1998;391:344-345.

38. Qu T, Yang H, Walston JD, Fedarko NS, Leng SX. Upregulated monocytic expression of CXC chemokine ligand 10 (CXCL-10) and its relationship with serum interleukin-6 levels in the syndrome of frailty. Cytokine. 2009;46:319-324.

39. Qu T, Walston JD, Yang H, et al. Upregulated ex vivo expression of stressresponsive inflammatory pathway genes by LPS-challenged CD14(+) monocytes in frail older adults. Mech Ageing Dev. 2009;130:161-166.

40. Schmaltz HN, Fried LP, Xue QL, Walston J, Leng SX, Semba RD. Chronic cytomegalovirus infection and inflammation are associated with prevalent frailty in community-dwelling older women. J Am Geriatr Soc. 2005;53:747-754.

41. Wang GC, Kao WH, Murakami P, et al. Cytomegalovirus infection and the risk of mortality and frailty in older women: a prospective observational cohort study. Am J Epidemiol. 2010;171:1144-1152.

42. Leng SX, Qu T, Semba RD, et al. Relationship between cytomegalovirus (CMV) IgG serology, detectable CMV DNA in peripheral monocytes, and CMV pp65(495-503)-specific CD8(+) T cells in older adults. Age (Dordr). 2011;33:607-614.

43. Leng SX, Li H, Xue QL, et al. Association of detectable cytomegalovirus (CMV) DNA in monocytes rather than positive CMV IgG serology with elevated neopterin levels in community-dwelling older adults. Exp Gerontol. 2011;46:679-684.
44. Visser M, Pahor M, Taaffe DR, et al. Relationship of interleukin-6 and tumor necrosis factor-alpha with muscle mass and muscle strength in elderly men and women: the Health ABC Study. J Gerontol A Biol Sci Med Sci. 2002;57:M326-M332.

45. Ferrucci L, Penninx BW, Volpato S, et al. Change in muscle strength explains accelerated decline of physical function in older women with high interleukin-6 serum levels. $J$ Am Geriatr Soc. 2002;50: 1947-1954.

46. Puts MT, Visser M, Twisk JW, Deeg DJ, Lips P. Endocrine and inflammatory markers as predictors of frailty. Clin Endocrinol (Oxf). 2005;63:403-411.

47. Chaves PH, Semba RD, Leng SX, et al. Impact of anemia and cardiovascular disease on frailty status of community-dwelling older women: the Women's Health and Aging Studies I and II. J Gerontol A Biol Sci Med Sci. 2005;60:729-735.

48. Newman AB, Gottdiener JS, Mcburnie MA, et al. Associations of subclinical cardiovascular disease with frailty. J Gerontol A Biol Sci Med Sci. 2001;56:M158-M166.

49. Leng SX, Cappola AR, Andersen RE, et al. Serum levels of insulin-like growth factor-I (IGF-I) and dehydroepiandrosterone sulfate (DHEA-S), and their relationships with serum interleukin-6, in the geriatric syndrome of frailty. Aging Clin Exp Res. 2004;16:153-157.

50. Leng SX, Hung W, Cappola AR, Yu Q, Xue QL, Fried LP. White blood cell counts, insulin-like growth factor-1 levels, and frailty in community-dwelling older women. J Gerontol A Biol Sci Med Sci. 2009;64:499-502.

51. Michelon E, Blaum C, Semba RD, Xue QL, Ricks MO, Fried LP. Vitamin and carotenoid status in older women: associations with the frailty syndrome. J Gerontol A Biol Sci Med Sci. 2006;61: 600-607.

52. Vasto S, Mocchegiani E, Malavolta M, et al. Zinc and inflammatory/ immune response in aging. Ann NY Acad Sci. 2007;1100:111-122.

53. Arai Y, Takayama M, Gondo Y, et al. Adipose endocrine function, insulin-like growth factor-1 axis, and exceptional survival beyond 100 years of age. J Gerontol A Biol Sci Med Sci. 2008;63:1209-1218.

54. Reiner AP, Aragaki AK, Gray SL, et al. Inflammation and thrombosis biomarkers and incident frailty in postmenopausal women. Am J Med. 2009;122:947-954.

55. LaCroix AZ, Gray SL, Aragaki A, et al. Statin use and incident frailty in women aged 65 years or older: prospective findings from the Women's Health Initiative Observational Study. J Gerontol A Biol Sci Med Sci. 2008;63:369-375.

56. Liu LK, Lee WJ, Liu CL, et al. Age-related skeletal muscle mass loss and physical performance in Taiwan: implications to diagnostic strategy of sarcopenia in Asia. Geriatr Gerontol Int. 2013;13: 964-971.

57. Rockwood K, Bergman H. FRAILTY: a report from the 3(rd) Joint Workshop of IAGG/WHO/SFGG, Athens, Jan 2012. Can Geriatr J. 2012;15:31-36.

58. Sternberg SA, Levin R, Dkaidek S, Edelman S, Resnick T, Menczel J. Frailty and osteoporosis in older women - a prospective study. Osteoporos Int. Epub September 4, 2013.

59. Varadhan R, Walston J, Cappola AR, Carlson MC, Wand GS, Fried LP. Higher levels and blunted diurnal variation of cortisol in frail older women. J Gerontol A Biol Sci Med Sci 2008;63:190-195.

60. Shardell M, Hicks GE, Miller RR, et al. Association of low vitamin D levels with the frailty syndrome in men and women. $J$ Gerontol A Biol Sci Med Sci. 2009;64:69-75.

61. Blaum CS, Xue QL, Michelon E, Semba RD, Fried LP. The association between obesity and the frailty syndrome in older women: the Women's Health and Aging Studies. J Am Geriatr Soc. 2005;53:927-934.

62. Woods NF, LaCroix AZ, Gray SL, et al. Frailty: emergence and consequences in women aged 65 and older in the Women's Health Initiative Observational Study. J Am Geriatr Soc. 2005;53:1321-1330.

63. Hubbard RE, Lang IA, Llewellyn DJ, Rockwood K. Frailty, body mass index, and abdominal obesity in older people. J Gerontol A Biol Sci Med Sci. 2010;65:377-381. 
64. Strandberg TE, Sirola J, Pitkala KH, Tilvis RS, Strandberg AY, Stenholm S. Association of midlife obesity and cardiovascular risk with old age frailty: a 26-year follow-up of initially healthy men. Int $J$ Obes (Lond). 2012;36:1153-1157.

65. Stenholm S, Guralnik JM, Bandinelli S, Ferrucci L. The prognostic value of repeated measures of lower extremity performance: should we measure more than once? J Gerontol A Biol Sci Med Sci. Epub November 23, 2013.

66. Strandberg TE, Pitkala KH. Frailty in elderly people. Lancet. 2007;369:1328-1329.

67. Makary MA, Segev DL, Pronovost PJ, et al. Frailty as a predictor of surgical outcomes in older patients. J Am Coll Surg. 2010;210: 901-908.

68. Ronning B, Wyller TB, Seljeflot I, et al. Frailty measures, inflammatory biomarkers and post-operative complications in older surgical patients. Age Ageing. 2010;39:758-761.

69. Afilalo J, Karunananthan S, Eisenberg MJ, Alexander KP, Bergman H. Role of frailty in patients with cardiovascular disease. Am J Cardiol. 2009;103:1616-1621.

70. Iqbal J, Denvir M, Gunn J. Frailty assessment in elderly people. Lancet. 2013;381:1985-1986.

71. Yao X, Hamilton RG, Weng NP, et al. Frailty is associated with impairment of vaccine-induced antibody response and increase in postvaccination influenza infection in community-dwelling older adults. Vaccine. 2011;29:5015-5021.

72. Ridda I, MacIntyre CR, Lindley R, et al. Immunological responses to pneumococcal vaccine in frail older people. Vaccine. 2009;27: $1628-1636$.

73. Hamaker ME, Jonker JM, de Rooij SE, Vos AG, Smorenburg CH, van Munster BC. Frailty screening methods for predicting outcome of a comprehensive geriatric assessment in elderly patients with cancer: a systematic review. Lancet Oncol. 2012;13:e437-e444.

74. Audisio RA, van LB. When reporting on older patients with cancer, frailty information is needed. Ann Surg Oncol. 2011;18:4-5.

75. Extermann M, Overcash J, Lyman GH, Parr J, Balducci L. Comorbidity and functional status are independent in older cancer patients. $J$ Clin Oncol. 1998;16:1582-1587.

76. Courtney-Brooks M, Tellawi AR, Scalici J, et al. Frailty: an outcome predictor for elderly gynecologic oncology patients. Gynecol Oncol. 2012;126:20-24.

77. Aaldriks AA, van der Geest LG, Giltay EJ, et al. Frailty and malnutrition predictive of mortality risk in older patients with advanced colorectal cancer receiving chemotherapy. J Geriatr Oncol. 2013;4:218-226.

78. Bennett JA, Winters-Stone KM, Dobek J, Nail LM. Frailty in older breast cancer survivors: age, prevalence, and associated factors. Oncol Nurs Forum. 2013;40:E126-E134.
79. Desquilbet L, Jacobson LP, Fried LP, et al. HIV-1 infection is associated with an earlier occurrence of a phenotype related to frailty. J Gerontol A Biol Sci Med Sci. 2007;62:1279-1286.

80. Desquilbet L, Margolick JB, Fried LP, et al. Relationship between a frailty-related phenotype and progressive deterioration of the immune system in HIV-infected men. J Acquir Immune Defic Syndr. 2009;50: 299-306.

81. Piggott DA, Muzaale AD, Mehta SH, et al. Frailty, HIV infection, and mortality in an aging cohort of injection drug users. PLoS One. 2013;8:e54910.

82. Boyle PA, Buchman AS, Wilson RS, Leurgans SE, Bennett DA. Physical frailty is associated with incident mild cognitive impairment in community-based older persons. J Am Geriatr Soc. 2010;58: $248-255$.

83. Buchman AS, Boyle PA, Wilson RS, Tang Y, Bennett DA. Frailty is associated with incident Alzheimer's disease and cognitive decline in the elderly. Psychosom Med. 2007;69:483-489.

84. Song X, Mitnitski A, Rockwood K. Nontraditional risk factors combine to predict Alzheimer disease and dementia. Neurology. 2011;77:227-234.

85. Theou O, Stathokostas L, Roland KP, et al. The effectiveness of exercise interventions for the management of frailty: a systematic review. J Aging Res. 2011;2011:569194.

86. Gill TM, Baker DI, Gottschalk M, Peduzzi PN, Allore H, Byers A. A program to prevent functional decline in physically frail, elderly persons who live at home. $N$ Engl J Med. 2002;347:1068-1074.

87. Fiatarone MA, O’Neill EF, Ryan ND, et al. Exercise training and nutritional supplementation for physical frailty in very elderly people. N Engl J Med. 1994;330:1769-1775.

88. Forster A, Lambley R, Hardy J, et al. Rehabilitation for older people in long-term care. Cochrane Database Syst Rev. 2009;(1):CD004294.

89. Clegg AP, Barber SE, Young JB, Forster A, Lliffe SJ. Do home-based exercise interventions improve outcomes for frail older people? Findings from a systemic review. Rev Clin Gerontol. 2012;22:68-78.

90. Basaria S, Coviello AD, Travison TG, et al. Adverse events associated with testosterone administration. N Engl J Med. 2010;363:109-122.

91. Rossouw JE, Anderson GL, Prentice RL, et al. Risks and benefits of estrogen plus progestin in healthy postmenopausal women: principal results From the Women's Health Initiative randomized controlled trial. JAMA. 2002;288:321-333.

92. Friedlander AL, Butterfield GE, Moynihan S, et al. One year of insulinlike growth factor I treatment does not affect bone density, body composition, or psychological measures in postmenopausal women. J Clin Endocrinol Metab. 2001;86:1496-1503.

93. Campbell S, Szoeke C. Pharmacological treatment of frailty in the elderly. J Pharm Pract Res. 2009;39:147-151.
Clinical Interventions in Aging

\section{Publish your work in this journal}

Clinical Interventions in Aging is an international, peer-reviewed journal focusing on evidence-based reports on the value or lack thereof of treatments intended to prevent or delay the onset of maladaptive correlates of aging in human beings. This journal is indexed on PubMed Central, MedLine, the American Chemical Society's 'Chemical Abstracts

\section{Dovepress}

Service' (CAS), Scopus and the Elsevier Bibliographic databases. The manuscript management system is completely online and includes a very quick and fair peer-review system, which is all easy to use. Visit http://www.dovepress.com/testimonials.php to read real quotes from published authors. 